Psychological Topics, 30 (2021), 1, 99-114

Original Scientific Paper

UDC: 159.97 .072

159.942 .072

https://doi.org/10.31820/pt.30.1.5

616.895 .4

\title{
Short-Term Effects of Fluctuations in Self-Esteem, Perceived Stress and Loneliness on Depressive States
}

\author{
Lisa Di Blas, Matteo Borella, and Donatella Ferrante \\ University of Trieste, Trieste, Italy
}

\begin{abstract}
Depression depends on risk factors such as loneliness, low self-esteem, and perceived stress when inter-individual differences are investigated in the long run. Depression, however, oscillates withinperson over short-time periods as well, but there is a lack of evidence on its temporary correlates. The present study explored how transitory feelings of depression covariate with states of loneliness, stress, and self-esteem at the within-person level, further inspecting how inter-individual differences contribute to understanding intra-individual dynamics. Seventy-four adults ( $M=33$ years) took part in the study and reported on daily depression, stress, loneliness, and self-esteem for eight successive evenings. The main results showed that within-person fluctuations in depression depended on transient changes in loneliness, self-esteem, and stress, with stress further moderating the depression-self-esteem association; the link between depression and its predictors was reciprocal; inter-individual differences in depression instability across the assessment occasions enhanced the effect of transitory loneliness on feelings of depression. The present findings revealed that withinperson associations for depression reflect correlation patterns between people, further highlighting how an individual's instability in depressive states is relevant for understanding who is more vulnerable to transitory depressive states, which might develop into trait-like conditions over longer time periods.
\end{abstract}

Keywords: depression, self-esteem, fluctuations, dynamics, diathesis-stress model

\section{Introduction}

Depression represents a common mood disorder worldwide, which, when is long-lasting and moderately to severely intense, seriously affects an individual's health and everyday life conditions, at worst leading to suicide. In addition to

Lisa Di Blas, Department of Life Sciences, University of Trieste, Via Weiss, 2 (pal Q), 34134 Trieste, Italy. E-mail: diblali@units.it

\section{Acknowledgements}

We are grateful to two anonymous reviewers for their thorough and helpful comments and suggestions.

Lisa Di Blas https://orcid.org/0000-0002-1374-2535 
biological vulnerabilities (Hankin, 2015) and cognitive-affective patterns (Brewin, 1996), numerous prospective risk factors for depression have been reliably identified, among which are stressful life events (Hammen, 2015), loneliness (Park et al., 2020), and self-esteem (Orth \& Robins, 2013). In the present study, we investigated how depression states change in relation to within-person fluctuations in loneliness, stress, and self-esteem.

Loneliness is rooted in the fundamental need to belong or be accepted and, as such, it is cross-cultural (Baumeister \& Leary, 1995; Dweck, 2017), it is experienced by both men and women (with mixed findings on whether women experience it more frequently or intensely in comparison to men), and across the life span (although negatively associated with age, being prevalent during adolescence, Heinrich \& Gullone, 2006). Loneliness arises when a sense of belongingness is insufficiently met (Baumeister \& Leary, 1995) and it depends on the perceived quality of social relationships (Wheeler et al., 1983) rather than on the number of social contacts and interpersonal daily activities (Hawkley et al., 2003). As such, loneliness represents a cognitive-affective experience, which arises when an individual perceives a discrepancy between actual and ideal social relationships (Asher \& Paquette, 2003). Such a conceptualization of loneliness makes it distinct from states of solitude or social deprivation. In addition to poorer social competencies, quality of interpersonal relationships, and physical health conditions such as eating disorders and drug consumption, several psychological variables correlate with loneliness (Heinrich \& Gullone, 2006; Park et al., 2020). Together with desperation and impatient boredom, depression represents a basic affective feature of loneliness (Rubenstain \& Shaver, 1982). As such, it substantially is associated with loneliness both concurrently and across time, at all ages. Specifically, longitudinal studies indicate that initial loneliness levels predict later increases in depression, and as a chronic individual characteristic, loneliness contributes to the development and maintenance of dysfunctional psychological profiles (Green et al., 1992; Park et al., 2020). Therefore, several approaches have been developed in order to understand the determinants of such an affective condition in the long run (Heinrich \& Gullone, 2006).

Loneliness is a common short-term experience as well, but within-person daily oscillations in loneliness and its covariations with affective or cognitive outcomes have not been extensively explored yet, though they might help clarify mechanisms leading to later relatively stable dysfunctional patterns. In accordance with this line of reasoning, attention has been especially focused on transient links between loneliness as state, and social contacts and relationships. For example, van Winkel and colleagues (2017) demonstrated the reciprocal influence of feelings of loneliness and frequency of social contacts, with decreases in interpersonal occasions favouring an individual's transition to a major depressive condition 20 months later, in a female general population sample (van Winkel et al., 2017). Mote and colleagues (2020) provided additional support on how negative appraisals in social interactions 
covariate with momentary feelings of loneliness, further demonstrating that the within-person association was stronger for more lonely people (Mote et al., 2020). Arpin et al. (2015) recently found that intensity in oscillations in momentary loneliness, i.e., its instability, predicts alcohol consumption in social vs. solitary contexts. To our knowledge, less is known on how loneliness and depression-like states covariate daily, from a within-person perspective.

Self-esteem as an individual's positive evaluation of the self and her/his worth is a well-established predictor of depression. Longitudinal studies have evidenced that self-esteem represents a risk factor for increases in depression, although they also show that higher depressive levels undermine self-esteem in the long run (Orth \& Robins, 2013; Sowislo \& Orth, 2013). The robust effect of self-esteem on depression does not depend on gender, age, or measures adopted for assessing the two variables (Sowislo \& Orth, 2013), and remains substantial after controlling for variables such as anxiety, neuroticism and the five-factor model domains, or narcissism (Orth et al., 2016; Sowislo et al., 2014). Furthermore, self-esteem moderates the trajectory of depression across periods of years (Orth et al., 2012). Lastly, rank-order stability studies on self-esteem indicate that such a self-view substantially is as stable as personality traits generally are (Trzesniewski et al., 2003). Nevertheless, daily fluctuations can be observed as well, and they are informative. For example, when daily oscillations in self-esteem are investigated, they reveal that adolescents whose self-esteem levels fluctuate more from day to day, on average also tend to refer higher negative affect, and lower positive affect and self-esteem (Nelis \& Bukowski, 2019). Overall, self-esteem instability contributes to predicting psychological outcomes over and above self-esteem mean levels (Altman \& Roth, 2018). However, these findings are from a between-people analytical approach. Again, currently available literature does not offer information on how within-person daily fluctuations in self-esteem and depression are associated with each other.

A further risk factor for the development of depressive conditions which has to be taken into account is subjective stress. Several models have been developed in order to understand the stress-depression link in deep. Among them, the diathesisstress paradigm of depression posits that stressful events strengthen the impact of other vulnerability factors such as genetic profiles, personality, and social relationships on depression (Colodro-Conde et al., 2018; Hammen, 2015). When Orth and colleagues regarded self-esteem as diathesis, they found mixed support in favour of a moderation effect of stress on the association between self-esteem and depression (Orth et al., 2009). They also investigated whether stress is a precursor of both self-esteem and depression, with self-esteem therefore mistakenly representing a vulnerability factor for depression, but no support emerged for such a model. More recent research is indicating that the association between stress and depression is likely reciprocal and more complex. For example, from a developmental perspective, both sensitization and amplification processes have been demonstrated as well as cascade effects (Hankin, 2015; Liu \& Alloy, 2010). Therefore, the stress-depression 
relationship needs to be analysed from a more dynamic perspective, on both long- as well as short-term periods, because dynamic transient vulnerabilities may turn into more stable risk profiles across time (Hammen, 2015; Hankinn, 2015). When daily fluctuations in stress are studied in relation to mood, findings indicate a direct association, with contingent perceived stress impacting negative mood on the same day, with participants with worse supportive relationships and self-esteem being more likely to experience decreases in psychological well-being in the subsequent days as well (DeLongis et al., 1988).

\section{Study Aims}

Cross-sectional, as well as longitudinal studies steadily support reciprocal associations between depression and self-esteem, loneliness, and stress, with mixed findings on the moderating role of perceived stress on the relationship between depression and self-esteem. Less is known on how they are linked to each other in everyday experiences, though short-term changes and covariations help understand mechanisms underlying changes, which are observable over longer time periods.

In the current daily assessment study, we inspected how within-person fluctuations in loneliness, self-esteem and stress are associated with momentary changes in depressive states, further controlling for temporary anxiety, because of the anxiety-depression comorbidity in clinical as well as non-clinical settings (Alloy et al., 1990), and for the effect of positive events on depressive mood (Oishi et al., 2007). In accordance with the literature on concurrent and longitudinal associations, we expected that each estimator uniquely accounts for fluctuations in depressive states (Hammen, 2015; Heinrich \& Gullone, 2006; Orth \& Robins, 2013), with perceived stress further moderating the impact of both self-esteem (Orth et al., 2009) and tentatively loneliness on depression. Furthermore, the hypothesis that the association between momentary depression and its predictors is reciprocal was investigated as well (Hankin, 2015; Liu \& Alloy, 2010).

We also analysed how more stable inter-individual differences moderate withinperson covariations between transitory experiences. In accordance with findings on daily self-esteem instability (Altman \& Roth, 2018; Nelis \& Bukowski, 2019), we included between-people differences in their depression instability and explored whether higher changeability across the days moderates within-person covariations.

\section{Method}

\section{Participants}

Seventy-four adults (33 males) took part in the present study. Their mean age was $33.45 \pm 14.25$ years, with no significant differences between males $(32.27 \pm$ 
13.66) and females $(34.39 \pm 14.80) ; 60.8 \%$ was younger than $30 ; 37.8 \%$ were university students.

\section{Materials and Procedure}

The participants were recruited by adopting convenience sampling. They were informed on the general aim of the study, on its procedure as well as on data treatment, privacy, and voluntary participation, in accordance with AIP ethical guidelines. Participants remained anonymous. Additional ethical approval was not required since there was no treatment including medical invasive diagnostics or procedures, which might cause psychological or social discomfort in the participants.

Once they accepted to take part in the study, the participants were asked to provide sex, age, whether they are students or workers; they then described how they felt or behaved in the last 3 months by using a 4-options Likert scale (from $1=n e v e r$ or rarely to $4=$ very often or always) along the items of the following questionnaires:

Generalized Anxiety Disorder or GAD-7. The scale is a free-to-use instrument and presents 7 items and assesses a generalized anxiety disorder (Spitzer et al., 2006); internal Cronbach's Alpha consistency for the present sample was .76.

Patient Health Questionnaire (PHQ-9). PHQ-9 is the depression module from the PRIME-MD screening instrument for most common mental disorders; the module presents 9 items and it is based on the diagnostic criteria of DSM-IV (Kroenke et al., 2001); for the present study we applied all the items but the last ("Thoughts that you would be better off dead, or of hurting yourself in some way?") because of its high clinical relevance; internal alpha consistency for the present sample was .76 (8 items).

Perceived Stress in Everyday Life (PSL). We assessed how much people felt stressed with their life by administering 4 items which were generated on the basis of the Perceived Occupational Stress scale (Marcatto et al., 2020); items such as My everyday life is stressful, or I feel under pressure for most of the day were administered; internal alpha consistency for the present sample was .74.

After filling out this first part which served as a baseline, the participants were asked to rate each evening, for eight successive days, how they had felt during that day, along with the DASS, UCLA, and RSES questionnaires; they started to provide daily self-ratings from the day after they had provided baseline self-descriptions. In all, we listed 41 items because we skipped few items among those listed in the questionnaires herein presented in order to avoid redundant sentences or clinically relevant contents, which were unfitting for the purposes of the present study; the same Likert scale was used for all the items, ranging from 1 (never today) to 4 (several times today). The questionnaires were as follows: 
The Rosenberg Self-Esteem Scale (RSES, Prezza et al., 1997, for the Italian version), a well-known self-report measure aimed at evaluating an individual's global self-worth (e.g. "I'm generally satisfied with me") by assessing both positive and negative feelings about oneself; 7 items were presented, and Cronbach's alphas, calculated for each assessment occasion, ranged from .84 to .90 .

The UCLA Loneliness Scale (UCLA, Boffo et al., 2012, for the Italian version), a 20-items questionnaire, tapping three main sub-components, i.e., Isolation, Relational connectedness, and a weaker general trait-like Loneliness experience (Boffo et al., 2012); for the present study, we presented 16 items (2 represented the trait-like Loneliness facet, 5 Isolation, and 9 represented the Relational connectedness facet) and calculated a general score only; alphas ranged from .89 to .92 across the eight assessment occasions.

The Depression Anxiety Stress Scales-21 (DASS-21, Bottesi et al., 2015, for the Italian version) is a brief scale aimed at assessing Depression, Stress, and Anxiety, 7 items for each domain originally, but for the present study we presented 6 items for Depression and 5 for Anxiety; alphas ranged from .83 to .93 for the DASS Stress scale, from .38 to .77 for Anxiety, and from .65 to .79 for Depression.

Each evening, the participants also reported on whether any positive or negative event, significant to them, had happened that day.

\section{Results}

We used a multilevel regression model in order to explore how depression, stress, loneliness, and self-esteem dynamically covariate across the assessment occasions, after controlling for anxiety and daily events, with the eight measurement occasions nested within-participants (Heck et al., 2010). The data consisted of 592 observations nested within 74 participants. Within-person or I level variables were centred around the mean of the single individual, in order to take under control between-people differences in the independent variables and inspect within-person variability only (Fleeson, 2007).

\section{Descriptive Statistics and Simple Correlations between Inter-Individual Differences in the Study Variables}

Mean scores were calculated for each participant for each of the study variables. Moreover, between-people differences in intensity of fluctuations, namely, instability in the study variables across the eight assessment occasions, were also calculated as the mean squared deviation from a participant's mean level of the respective variable (Nelis \& Bukowski, 2019). Table 1 presents descriptive statistics for and inter-correlations among the study variables. Mean levels indicate that the participants referred that they felt anxiety, depression or harass sometimes, while 


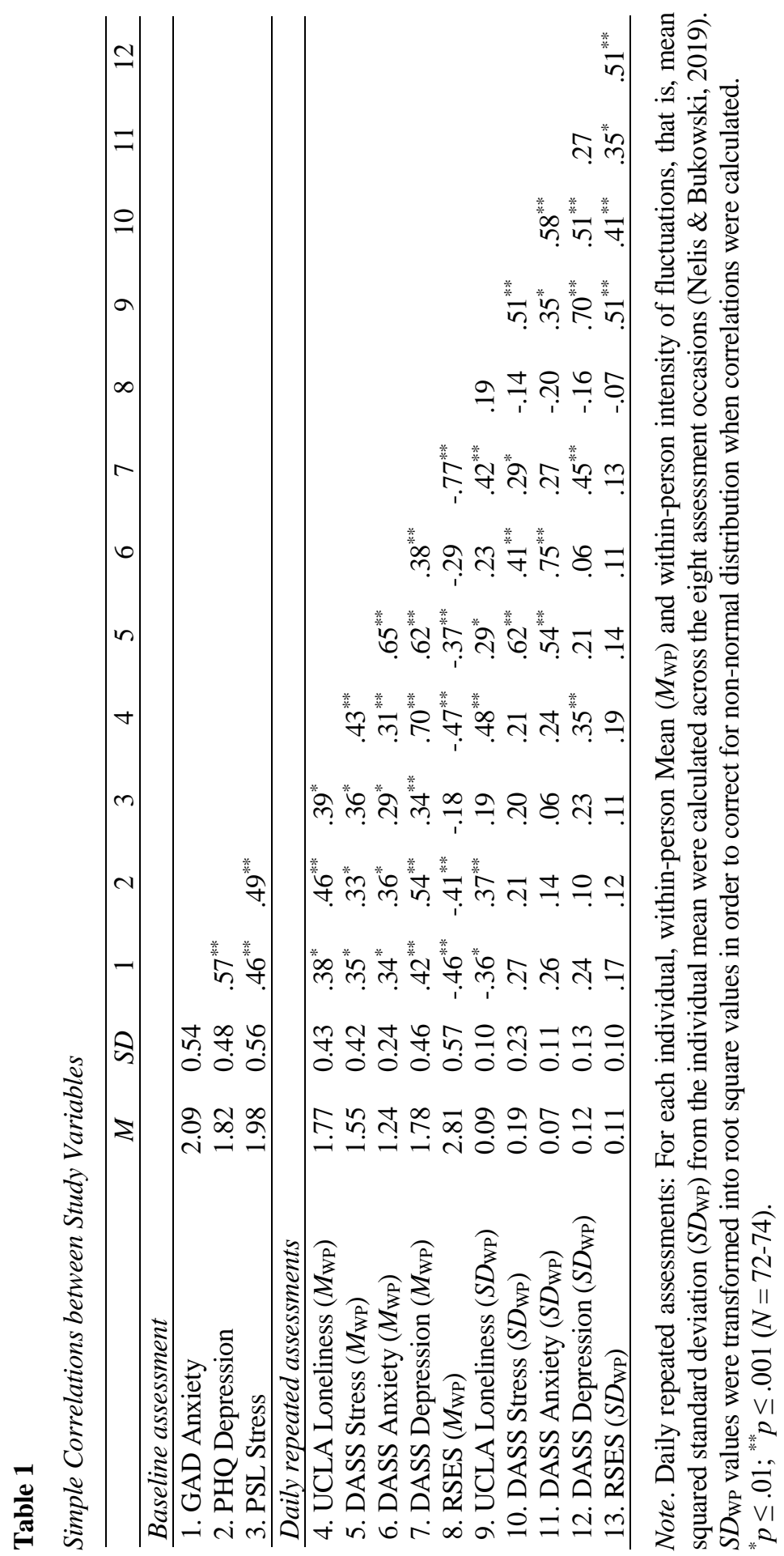


reporting to feel self-worth rather often; that is, overall, the participants referred they daily experienced emotional stability states and positive self-views. Descriptive statistics for variables instability across the eight days (i.e., $S D_{\mathrm{WP}}$ ) indicated contained oscillations intensity. Simple correlations showed that baseline self-rated PHQ Depression, GAD Anxiety, and PSL Stress were positively, but moderately associated with the aggregated scores on the DASS Depression, Anxiety, and Stress scales, respectively, thus indicating that baseline variables were convergent operationalizations of the same hypothesized mid-level constructs repeatedly assessed across the eight evenings.

Generally speaking, correlations among the study variables went in the expected direction, with depression being positively associated with loneliness, stress, anxiety, and low self-esteem. As to individual differences in intensity in within-person fluctuations across the eight days, the results showed that higher changeability in DASS Depression was positively associated with higher mean levels in DASS Depression; similarly, more lonely, anxious, or distressed people also revealed higher changeability in the corresponding domains, whereas self-esteem instability and mean levels did not correlate.

Any significant association did not emerge between sex and age (but $r=-.38, p$ $\leq 0.01$, for DASS Anxiety ${ }_{\text {Mwp }}$ ) and the study variables.

\section{Within-Person Covariations}

Table 2 reports on how within-person fluctuations in loneliness, stress, and selfesteem predicted depressive mood. The results for fixed effects showed how each estimator uniquely accounted for variability in DASS Depression; that is, when an individual increased, for example, her/his loneliness state of a single unit above her/his own loneliness mean score, $\mathrm{s} /$ he was expected to increase her/his depressive state of .27 point as well. Intercept random effects indicate variability across individuals in the outcome variable and therefore they are generally expected. Slope random effects indicate whether the association as fixed effect between the dependent variable and its predictor varies in any way in intensity among the individuals. The present results showed significant slope random effects for UCLA Loneliness and RSES Self-esteem. Reported as standard deviations, these effects indicated that transitory decreases in RSES Self-esteem and increases in depression positively covaried with momentary increases in depression for the majority of the participants, with random error for RSES Self-esteem $(b=-0.36 \pm 0.23)$, for example, indicating an expected $b \leq-0.13$ for approximately the $84 \%$ of the participants. When interaction terms, with DASS Stress as moderator, were added, the results showed that the within-person association between DASS Depression and RSES Self-esteem was stronger, if momentary perceived stress was higher (Table 2). The inclusion of the I level interaction term in the model enhanced the model fit significantly $(\Delta-2 \mathrm{LL}$ $=7.35, d f=1, p \leq .01)$. No further I level interaction effects emerged. 
Table 2 also shows that transitory changes in UCLA Loneliness were uniquely associated with intra-individual changes in RSES Self-esteem as well as in all the DASS variables; positive events additionally contributed to predict lower feelings of loneliness; any I level interaction effect did not emerge. Similarly, temporary RSES Self-esteem decreases depended on momentary within-person increases in DASS Depressive mood especially (with $b=-0.46 \pm 0.29$, that is $b \leq-0.17$ for $84 \%$ of the participants) and feelings of UCLA Loneliness, whereas a positive event helped selfworth; no interaction effect was significant. Lastly, in addition to momentary DASS Anxiety, within-person variability in DASS Depressive and UCLA Loneliness states uniquely predicted feeling of DASS Stress scores; unexpectedly, daily positive event also enhanced perceived stress. Overall, the results in Table 2 indicate that the associations among transient depression, self-esteem, and loneliness states are reciprocal, with stress enhancing the effect of loneliness on depression only.

\section{Table 2}

Within-Person Covariation in Transitory Changes in the Study Variables

\begin{tabular}{|c|c|c|}
\hline & Fixed effect $(b)$ & Random effect $(S D)$ \\
\hline & \multicolumn{2}{|c|}{ DASS Depression } \\
\hline Intercept & $1.77^{* * *}$ & $0.46^{* * *}$ \\
\hline UCLA Loneliness & $0.27^{* * *}$ & $0.30^{* *}$ \\
\hline RSES Self-esteem & $-.036^{* * *}$ & $0.23^{*}$ \\
\hline DASS Stress & $0.20^{* * *}$ & \\
\hline \multirow[t]{2}{*}{ DASS Stress by RSES } & $-0.21^{* * *}$ & \\
\hline & \multicolumn{2}{|c|}{ UCLA Loneliness } \\
\hline Intercept & $1.78^{* * *}$ & $0.42^{* * *}$ \\
\hline RSES Self-esteem & $-0.18^{* * *}$ & \\
\hline DASS Depression & $0.28^{* * *}$ & $0.25^{* *}$ \\
\hline DASS Stress & $0.14^{* * *}$ & \\
\hline DASS Anxiety & $0.12^{*}$ & \\
\hline \multirow[t]{2}{*}{ Daily event } & $-0.09^{* *}$ & \\
\hline & \multicolumn{2}{|c|}{ DASS Stress } \\
\hline Intercept & $1.56^{* * *}$ & $0.41^{* * *}$ \\
\hline UCLA Loneliness & $0.24^{* *}$ & $0.41^{* *}$ \\
\hline RSES Self-esteem & -0.02 & $0.34^{* *}$ \\
\hline DASS Depression & $0.33^{* * *}$ & \\
\hline DASS Anxiety & $0.67^{* * *}$ & $0.43^{* * *}$ \\
\hline \multirow{2}{*}{ Daily event } & $0.12^{* * *}$ & \\
\hline & \multicolumn{2}{|c|}{ RSES Self-esteem } \\
\hline Intercept & $2.81^{* * *}$ & $0.56^{* * *}$ \\
\hline UCLA Loneliness & $-0.33^{* * *}$ & \\
\hline DASS Depression & $-0.46^{* * *}$ & $0.29^{*}$ \\
\hline DASS Anxiety & $0.11^{*}$ & \\
\hline Daily event & $0.07^{* *}$ & \\
\hline
\end{tabular}

Note. Daily event coding: 1 = positive event, $0=$ negative event or no relevant event at all. I level independent variables were centred within-person around the individual's mean. ${ }^{*} p \leq .05 ;{ }^{* *} p \leq .01$; ${ }^{* * *} p \leq .001$. 


\section{Cross-Level Interaction Effects}

Intercept random effect for DASS Depression (Table 2) indicated significant inter-individual differences in this outcome variable, and simple correlations in Table 1 already suggested predictors of such variability. In addition to reduce the intercept random effect for DASS Depression, II level estimators can account for slope random effects as well, when they significantly interact with I level variables. For the present study, we focused on DASS Depression as an outcome and explored how inter-individual differences in the study variables, including sex and age, accounted for its variability; among predictors we included PHD Depression, GAD Anxiety, and PSL Stress instead of the mean scores on the DASS scales, so as to reduce common method variance, and included inter-individual differences in instability levels for both DASS and RSES variables ( $S D_{\mathrm{WP}}$, see Table 1). Table 3 presents the final model, after excluding statistically irrelevant predictors, and shows that RSES Self-esteem and DASS Stress interacted at within-person as well as at betweenpeople levels. In addition, higher DASS Depression instability predicted higher mean scores in DASS Depression, further interacting with within-person fluctuations in feelings of loneliness. This cross-level interaction effect improved the model fit significantly $(\Delta-2 \mathrm{LL}=12.56, d f=1, p \leq .001)$, reduced of $22 \%$ the slope random effect of UCLA Loneliness, and revealed that intra-individual changes in loneliness lead to sadness, especially in those people who fluctuate more than others around their average depression level.

\section{Table 3}

Predicting Transitory Depressive States from Both Intra- and Inter-Individual Differences

\begin{tabular}{lcc}
\hline & $\begin{array}{c}\text { Fixed effect } \\
(b)\end{array}$ & $\begin{array}{c}\text { Random effect } \\
(S D)\end{array}$ \\
\hline Intercept & 0.82 & $0.23^{* * *}$ \\
\hline I level variables wP & & \\
\hline UCLA Loneliness wP & -0.10 & $0.23^{*}$ \\
DASS Stress wP & $0.18^{* * *}$ & \\
RSES Self-esteem WP & $-0.35^{* * *}$ & \\
RSES Self-esteem wP by DASS Stress WP & $-0.16^{* *}$ & \\
\hline II level variables & & \\
\hline RSES Self-esteem & 0.11 \\
PSL Perceived Stress & $1.05^{* * *}$ \\
DASS Depression Instability $\left(S D_{\mathrm{WP}}\right)$ & $0.96^{* * *}$ & \\
RSES Self-esteem by PSL Stress & $-0.31^{* * *}$ & \\
\hline Cross level interaction effect & & \\
\hline UCLA Loneliness wP by DASS Depression $\left(\mathrm{SD}_{\mathrm{WP}}\right)$ & $1.11^{* * *}$ & \\
\hline
\end{tabular}

Note. $S D_{\mathrm{WP}}=$ Inter-individual instability levels or within-person intensity of fluctuations as mean squared standard deviation from the individual mean; $\mathrm{wP}=$ within-person. ${ }^{*} p \leq .05,{ }^{* *} p \leq .01,{ }^{* * *} p \leq .001$. 


\section{Discussion}

The present study explored the correlates of transitory experiences of depression at the within-person level. The results showed that daily fluctuations in loneliness, self-esteem, and stress accounted for depressive states, after controlling for anxiety, which did not account for additional variance. Such results are in line with the literature, demonstrating that inter-individual differences in trait-like loneliness, selfesteem and subjective stress contribute, each uniquely, to predict depression both concurrently and longitudinally (Hankin, 2015; Heinrich \& Gullone, 2006; Orth \& Robins, 2013). The present findings, therefore, cover a gap in the current research and reveal that intra-individual dynamics of transitory depression reflect betweenpeople association patterns of depression as a more stable individual characteristic. In addition, the present study showed that stress strengthened the effect of low selfesteem, but not of loneliness, on transitory depression, thus replicating the diathesis (as self-esteem)-stress model at intra-individual level (Hammen, 2015; Liu \& Alloy, 2010). The same interaction effect emerged at between-people level in our sample as well, with perceived stress as II level variable corresponding to a baseline selfevaluation provided along a different questionnaire from the one applied for daily self-ratings of stress. Overall, the moderation effect of stress on the association between depression and self-worth robustly emerged from the present data set. Sex or age did not moderate the association patterns we observed.

Daily positive events also impacted fluctuations in all the study variables, but depression. The general finding that daily events, when positive, help psychological well-being has been established cross-culturally as well (Oishi et al., 2007). Conversely, findings on the moderating role of stress in the association between daily events and outcome are more mixed (Carney et al., 2000); we did not find any empirical support to any interaction effect between daily positive events and the study predictors in the models here inspected.

Several models have been investigated in order to understand the association between depression and its major risk factors. Longitudinal studies have demonstrated that the relationship often is reciprocal, with self-esteem, for example, favouring increases in depressive levels, but depression eroding self-esteem in the long run as well (Orth \& Robins, 2013). We found that daily fluctuations in the study variables are reciprocal as well. We might, therefore, hypothesize that transitory changes in any of the variables here repeatedly assessed might alter an individual's current system and rapidly turn the person into different dynamics and mood, also thanks to the effect of positive events.

A further substantial finding of the present study is that inter-individual differences matter for a deeper understanding of an individual's dynamic functioning. Specifically, we found that the effect of momentary loneliness on transitory experiences of depression was stronger in those participants with higher depression instability levels across eight days. In other words, feeling lonely favours 
depression, especially in those people who report daily depression levels, which substantially oscillate from day to day. Such a cross-level interaction effect needs to be replicated, nevertheless, it represents a significant contribution. Firstly, it provides additional support to the idea of investigating systematically a person's variability in its momentary self-evaluations, because it reveals more stable psychological characteristics (Nelis \& Bukowski, 2019), i.e., between people differences, which in turn impact within-person dynamic changes over and above averaged scores across measurement occasions. Secondly, such an interaction effect generally highlights the advantage of adopting a multi-level approach for predicting as well as comprehending within-person functioning, thanks to the combination of withinperson and between-persons approaches, which represent two complementary rather than antagonistic perspectives to each other (Di Blas et al., 2017).

In brief, the present study offers support to traditional models of depression by inspecting its daily fluctuations and its risk factors, further supporting the diathesisstress model as a possible mechanism underlying dynamic processes as well. It further evidences the relevance of combining within- and between-persons approaches in order to predict an individual's dynamic patterns of depressive mood and suggests that positive events might help reduce a momentary discomfort as well. To our knowledge, our study presents some new findings, which need to be replicated.

Lastly, we acknowledge several limits. Methodologically, the participants provided self-ratings for eight consecutive evenings only, so we could not explore how the study variables covariate at a different time of the day nor the effect of the time on their covariations. Moreover, we did not systematically take under control the day of the week, although it has been demonstrated that week-ends favour increases in positive mood (Egloff et al., 1995). Generally, such a limit did not allow us to explore the week trend of the study variables as well as to take under control its effect and detrend the data (Wang \& Maxwell, 2015). A further methodological limit deals with the use of shortened questionnaires for daily assessments. Although we carefully excluded some of those items which are very similar in content to each other, in order to limit repetitions, or those items which are clinically relevant and therefore inadequate for a daily self-report in non-clinical participants, we acknowledge that such omissions can partially alter the underlying construct and the results thereby.

Secondly, we did not include any measure of social contacts, although they represent a relevant risk factor for both depression and loneliness as states or as traitlike characteristics (Steger \& Kashdan, 2009). As to daily events, they were here assessed as a dichotomous variable only, positive or not, and remained unspecified in their content. They instead deserve more attention in the dynamic processes such as those here investigated, also in interaction with between-people differences (Carney et al., 2000). We also put marginal attention on anxiety and mostly aimed to take it under control, because it generally accompanies depression (Tiller, 2013). The 
present results however revealed that it did not contribute to account for withinperson fluctuations in daily depression, although it was substantially correlated with depression at between-people level. Nevertheless, reliability levels for daily DASS Anxiety assessments were critical and thereby the present results need to be cautiously interpreted. Lastly, the present findings suggest how daily dynamics work in non-clinical people, who exhibited contained intensity of fluctuations; clinical samples should be involved as well. Future studies might overcome such limits.

\section{References}

Alloy, L. B., Kelly, K. A., Mineka, S., \& Clements, C. M. (1990). Comorbidity of anxiety and depressive disorders: A helplessness-hopelessness perspective. In J. D. Maser \& C. R. Cloninger (Eds.), Comorbidity of mood and anxiety disorders (pp. 499-543). American Psychiatric Association.

Altmann, T., \& Roth, M. (2018). The Self-esteem Stability Scale (SESS) for cross-sectional direct assessment of Self-esteem stability. Frontiers in Psychology, 9, 91-100. https://doi.org/10.3389/fpsyg.2018.00091

Arpin, S. N., Mohr, C. D., \& Brannan, D. (2015). Having friends and feeling lonely: A daily process examination of transient loneliness, socialization, and drinking behavior. Personality and Social Psychology Bulletin, 41, 615-628. https://doi.org/10.1177/ 0146167215569722

Asher, S. R., \& Paquette, J. A. (2003). Loneliness and peer relations in childhood. Current Directions in Psychological Science, 12, 75-78.

Baumeister, R. F., \& Leary, M. R. (1995). The need to belong: Desire for interpersonal attachments as a fundamental human motivation. Psychological Bulletin, 117, 497-529.

Boffo, M., Mannarini, S., \& Munari, C. (2012). Exploratory structure equation modelling of the UCLA Loneliness Scale: A contribution to the Italian adaptation. TPM Testing Psicometria Metodologia, 19, 345-363.

Bottesi, G., Ghisi, M., Altoè, G., Conforti, E., Melli, G., \& Sica, C. (2015). The Italian version of the Depression Anxiety Stress Scales-21: Factor structure and psychometric properties on community and clinical samples. Comprehensive Psychiatry, 60, 170181. https://doi.org/10.1016/j.comppsych.2015.04.005

Brewin, C. R. (1996). Theoretical foundations of cognitive-behavior therapy for anxiety and depression. Annual Review of Psychology, 47, 33-57. https://doi.org/10.1146/annurev. psych.47.1.33

Carney, M. A., Armeli, S., Tennen, H., Affleck, G., \& O’Neil, T. P. (2000). Positive and negative daily events, perceived stress, and alcohol use: A diary study. Journal of Consulting and Clinical Psychology, 68, 788-798. https://doi.org/10.1037/0022006X.68.5.788 
Colodro-Conde, L., Couvy-Duchesne, B., Zhu, G., Coventry, W. L., Byrne, E. M., Gordon, S., Wright, M. J., Montgomery, G. W., Madden, P. A. F., Major Depressive Disorder Working Group of the Psychiatric Genomics Consortium, Ripke, S., Eaves, L. J., Heath, A. C., Wray, N. R., Medland, S. E., \& Martin, N. G. (2018). A direct test of the diathesisstress model for depression. Molecular Psychiatry, 23, 1590-1596. https://doi.org/10. 1038/mp.2017.130

DeLongis A., Folkman S., \& Lazarus R. S. (1988). The impact of daily stress on health and mood: Psychological and social resources as mediators. Journal of Personality and Social Psychology, 54, 486-495. https://doi.org/10.1037//0022-3514.54.3.486

Di Blas, L., Grassi, M., Carnaghi, A., Ferrante, D., \& Calarco, D. (2017). Within-person and between-people variability in personality dynamics: Knowledge structures, selfefficacy, pleasure appraisals, and the Big Five. Journal of Research in Personality, 70, 84-92. https://doi.org/10.1016/j.jrp.2017.06.002

Dweck, C. S. (2017). From needs to goals and representations: Foundations for a unified theory of motivation, personality, and development. Psychological Review, 124, 689719. https://doi.org/10.1037/rev0000082

Egloff, B., Tausch, A., Kohlmann, C. W., \& Krohne, H. W. (1995). Relationships between time of day, day of the week, and positive mood: Exploring the role of the mood measure. Motivation and Emotion, 19, 99-110. https://doi.org/10.1007/BF02250565

Fleeson, W. (2007). Studying personality processes: Explaining change in between-persons longitudinal and within-person multilevel models. In R. W. Robins, R. C. Fraley, \& R. F. Krueger (Eds.), Handbook of research methods in personality psychology (pp. 523542). The Guilford Press.

Green, B. H., Copeland, J. R. M., Dewey, M. F., Sharma, V., Saunders, P. A., Davidson, L. A., Sullivan, C., \& McWilliam, C. (1992). Risk factors for depression in elderly people: A prospective study. Acta Psychiatrica Scandinavica, 86, 213-217. https://doi.org/ 10. 1111/j.1600-0447.1992.tb03254.x

Hammen, C. L. (2015). Stress and depression: Old questions, new approaches. Current Opinion in Psychology, 4, 80-85. https://doi.org/10.1016/j.copsyc.2014.12.024

Hankin, B. M. (2015). Depression from childhood through adolescence: Risk mechanisms across multiple systems and levels of analysis. Current Opinion in Psychology, 4, 1320. https://doi.org/10.1016/j.copsyc.2015.01.003

Hawkley, L. C., Burleson, M. H., Berntson, G. G., \& Cacioppo, J. T. (2003). Loneliness in everyday life: Cardiovascular activity, psychosocial context, and health behaviors. Journal of Personality and Social Psychology, 85, 105-120.

Heck, R. H., Thomas, S. L., \& Tabata, L. N. (2010). Multilevel and longitudinal modelling with IBM SPSS. Taylor \& Francis Group.

Heinrich, L. M., \& Gullone, E. (2006). The clinical significance of loneliness: A literature review. Clinical Psychology Review, 26, 695-718. https://doi:10.1016/j.cpr.2006.04.002

Kroenke, K., Spitzer, R. L., \& Williams, J. B. (2001). The PHQ-9: Validity of a brief depression severity measure. Journal of General Internal Medicine, 16, 606-613. 
Liu, R. T., \& Alloy, L. B. (2010). Stress generation in depression: A systematic review of the empirical literature and recommendations for future study. Clinical Psychology Review, 30, 582-593. https://doi.org/10.1016/j.cpr.2010.04.010

Marcatto, F., Di Blas, L., Luis, O., Festa, S., \& Ferrante, D. (2020). The Perceived Occupational Stress scale: A brief tool for measuring workers' perception of stress at work. Manuscript submitted for publication.

Mote, J., Gonzalez, R., Kircos, C., Gard, D. E., \& Fulford, D. (2020). The relationship between state and trait loneliness and social experiences in daily life. PsyArXive Preprints. https://doi.org/10.31234/osf.io/pr79g

Nelis, S., \& Bukowski, W. M. (2019). Daily affect and self-esteem in early adolescence: Correlates of mean levels and within-person variability. Psychologica Belgica, 59, 96 115. https://doi.org/10.5334/pb.467

Oishi S., Diener, E., Choi, D. W., Kim-Prieto, C., \& Choi, I. (2007). The dynamics of daily events and well-being across cultures: When less is more. Journal of Personality and Social Psychology, 93, 685-698. https://doi.org/10.1037/0022-3514.93.4.685

Orth, U., \& Robins, R. W. (2013). Understanding the link between low self-esteem and depression. Current Directions in Psychological Science, 22, 455-460. https://doi.org/ $10.1177 / 0963721413492763$

Orth, U., Robins, R. W., \& Meier, L. L. (2009). Disentangling the effects of low self-esteem and stressful events on depression: Findings from three longitudinal studies. Journal of Personality and Social Psychology, 97, 307-321. https://doi.org/10.1037/a0015645

Orth, U., Robins, R. W., Meier, L. L., \& Conger, R. D. (2016). Refining the vulnerability model of low self-esteem and depression: Disentangling the effects of genuine selfesteem and narcissism. Journal of Personality and Social Psychology, 110, 133-149. https://doi.org/10.1037/pspp0000038

Orth, U., Robins, R. W., \& Widaman, K. F. (2012). Life-span development of self-esteem and its effects on important life outcomes. Journal of Personality and Social Psychology, 102, 1271-1288. https://doi.org/10.1037/a0025558

Park, C., Majeed, A., Gill, H., Tamura, J., Ho, R. C., Mansur, R. B., Nasri, F., Lee, Y., Rosenblat, J. D., Wong, E., \& McIntyre, R. S. (2020). The effect of loneliness on distinct health outcomes: A comprehensive review and meta-analysis. Psychiatry Research, 294, 113514. https://doi.org/10.1016/j.psychres.2020.113514

Prezza, N., Trombaccia, F. R., \& Armento, L. (1997). La scala dell'autostima di Rosenberg. Traduzione e validazione italiana [Rosenberg's self-esteem scale. Italian translation and validation]. Bollettino di Psicologia Applicata, 223, 35-44.

Rubenstein, C., \& Shaver, P. (1982). The experience of loneliness. In L. A. Peplau \& D. Perlman (Eds.), Loneliness: A sourcebook of current theory, research and therapy (pp. 206-223). Wiley.

Sowislo, J. F., \& Orth, U. (2013). Does low self-esteem predict depression and anxiety? A meta-analysis of longitudinal studies. Psychological Bulletin, 139, 213-240. https://doi.org/10.1037/a0028931 
Sowislo, J. F., Orth, U., \& Meier, L. L. (2014). What constitutes vulnerable self-esteem? Comparing the prospective effects of low, unstable, and contingent self-esteem on depressive symptoms. Journal of Abnormal Psychology, 123, 737-753.

Spitzer, R. L., Kroenke, K., Williams, J. B., \& Löwe, B. (2006). A brief measure for assessing generalized anxiety disorder. The GAD-7. Archives of Internal Medicine, 166, 10921097.

Steger, M. F., \& Kashdan, T. B. (2009). Depression and everyday social activity, belonging, and well-being. Journal of Counseling Psychology, 56(2), 289-300. https://doi.org/10. $1037 / \mathrm{a} 0015416$

Tiller, J. W. (2013). Depression and anxiety. The Medical Journal of Australia, 199, S28-31. https://doi.org/10.5694/mja12.10628

Trzesniewski, K. H., Donnellan, M. B., Robins, R. W. (2003). Stability of self-esteem across the life span. Journal of Personality and Social Psychology, 84, 205-220.

van Winkel, M., Wichers, M., Collip, D., Jacobs, N., Derom, C., Thiery, E., Myin-Germeys, I., \& Peeters, F. (2017). Unraveling the role of loneliness in depression: The relationship between daily life experience and behavior. Psychiatry, 80, 104-117, https://doi.org/10. 1080/00332747.2016.1256143

Wang, L. P., \& Maxwell, S. E. (2015). On disaggregating between-person and within-person effects with longitudinal data using multilevel models. Psychological Methods, 20, 6383. https://doi.org/10.1037/met0000030

Wheeler, L., Reis, H., \& Nezlek, J. B. (1983). Loneliness, social integration, and sex roles. Journal of Personality and Social Psychology, 45, 943-953.

Received: January 15, 2021 